\title{
Design and implementation of uniform light guide based, force and deflection measurement device
}

\author{
V. Ostaševičius*, P. Karpavičius**, G. Janušas***, G. Balevičius**** \\ *Institute of Mechatronics, Kaunas University of Technology, Kaunas, LT-44244, Lithuania, \\ E-mail: vytautas.ostasevicius@ktu.lt \\ **Department of Mechanical Engineering, Kaunas University of Technology, Kaunas, LT - 44244, Lithuania, \\ E-mail: karpaviciuspaulius@gmail.com \\ ***Department of Mechanical Engineering, Kaunas University of Technology, Kaunas, LT - 44244, Lithuania, \\ E-mail: Giedrius.janusas@ktu.lt \\ ****Institute of Mechatronics, Kaunas University of Technology, Kaunas, LT-44244, Lithuania, \\ E-mail: gytautasbalevicius@gmail.com
}

cross $^{\text {ref }}$ http://dx.doi.org/10.5755/j01.mech.22.4.15414

\section{Introduction}

Seamless application of sensors in the manufacturing environment as well as their subsequent integration into cloud computing capabilities is an indistinguishable part of the new cloud manufacturing paradigm $[1,2]$. Investigation of more easily applicable and universal novel sensing solutions is an endeavor of paramount importance to progress of many manufacturing industries.

Optical sensing devices have found countless applications in different industries, ranging from manufacturing [3], to automotive [4] to construction [5] and aerospace industries [6]. A high share of optical sensing in industry devices is attributable to the use of fiber optics based sensors [7].

These devices seem to be a mainstay of the industry, mainly due to their relatively high availability and low price, when compared to electromechanical sensors. Besides the already mentioned benefits of using fiber optics sensors, additional benefits, when compared to their electromechanical counterparts are: light weight, small size, immunity to electromagnetic interference, can sustain environmental vibration and shock, have high sensitivity and usually they are fully-dielectric [7].

Optical sensing devices using fiber optics have been around for over 40 years, beginning from photonic sensor patented in 1960s that was based on bifurcated fiber bundles with half the bundle used to illuminate a surface ant the reflection from the surface received by the other half of the bundle. After calibration the received signal allowed a very precise indication of the relative position of the end and the reflecting surface. After that the technology evolved with different approaches by the following sequence as given here [8]:

1) All Fiber Mach-Zehnder Interferometer.

2) All Fiber Michelson Interferometer.

3) Interferometric Multiplexing.

4) Sagnac Interferometer.

5) Fiber Bragg grating.

In the recent years there has been a high interest in applying structural health monitoring (SHM) techniques in various types of constructions. This need for SHM, has escalated due to increased use of pre-stressed concrete, specifically in bridge building. In fact around $44 \%$ of all new bridges built in US from 2009 and 2010 were made of pre- stressed concrete [9]. The use of pre-stressed concrete necessitates for a better assessment of its structural behavior [10].

SHM usually is a continuous method of monitoring structures performance and health condition. Where a sensor network, of optical fiber sensors is used by embedding it inside concrete cross-sections of pre-stressed beams or other parts of the structure under investigation [10].

Optical fiber sensors in this approach are used to capture pre-stressing forces at extremities of the beam structure, as applied to the structure and locations of maximum positive and negative bending moments, to capture forces at the most loaded locations [10].

Currently for SHM monitoring in structures (primarily bridges) the most widespread type fiber optics sensor is SOFO, a displacement sensor with a resolution in the micrometer range, developed at the Swiss Federal Institute of Technology in Laussane [5].

SOFO measurement setup uses low-coherence interferometry to measure the length difference between two optical fibers installed on the structure to be monitored. The measurement fiber is pretension and mechanically coupled to the structure at two anchorage points in order to follow its deformations, while the reference fiber is free and acts as a temperature reference. Both fibers are installed inside the same pipe and the measurement basis can be chosen between $200 \mathrm{~mm}$ and $10 \mathrm{~mm}$. The resolution of the system is of $2 \mu \mathrm{m}$ independently from the measurement basis and its precision of $0.2 \%$ of the measured deformation even over years of operation [5].

SOFO fiber optics sensor is the most widely used sensor of this type in Europe with more than a thousand units installed to this day and counting [5].

Besides that the following fiber optic sensors for civil structure monitoring are used in Europe [5]:

1) Microbend.

2) Bragg grating.

3) Fabry-Perot.

4) Raman.

5) Brillouin.

6) Hydrogen.

Due to the increase in usage of SHM for structure health control the need for simple, cheap and easy to assemble optical sensors is more prevalent than ever before.

Besides using optical sensors for SHM monitoring, 
there has been some research done in search of other options as:

1) High-speed CW step-frequency coherent radar for dynamic monitoring of civil engineering structures. The radar operated a continuous-wave stepfrequency in Ku-band, and the base-band signal is generated by direct digital synthesis [11].

2) A cement based piezoelectric composite sensor using 1-3 cement based piezoelectric composite as sensing element [12].

3) Structure monitoring using image stations.

Though most of the sensors or systems mentioned above are in research stage thus the main option for structure SHM monitoring is to use fiber optics sensors.

For this reason it was decided to investigate the possibility to use light guide based optical sensor keeping its application for SHM monitoring in mind. The main benefits observed in investigating applicability of such approach are the sensors low cost, high level of design customization and its ease of use and assembly which satisfies the needs pointed out before. The device should consist of a light source (preferably coherent to reduce uncertainties in the measurement results due to different wavelength of from the source), a translucent material part - light guide where the light would be guided through and any effect on the light guide (environmental forces) would affect the light guides geometry and thus reducing or increasing the amount of light transmitted through it. Last part of the light guide optical device for force or deformation measurements should include a light collector that would enable to track the change of transmitted light through the light guide.

Though the focus here is to see the light guide based optical device for force and deflection forces in the SHM monitoring field, nonetheless sensor has possible applicability in manufacturing or automotive, where robust, simple and economically viable sensor for force and deflection sensing is needed.

Special interest should be taken into possible application of the device in the field of biomechanics-biomedicine-bio tactile due to the sensors availability to be scaled down based on the necessary measurement range and sensitivity, and the available range of translucent materials for light guide depending on the aggressiveness of the environment the senor would be used in.

Light guide based force and deflection device could be considered as a substitute or enhancement for currently available Fiber Bragg Grating Force sensor used in minimal invasive surgery or any biological applications for force measurements where electromagnetic interference is present for a reliable, simple, accurate force or deflection measurement device. [13, 14].

In this article will be presented preoperational work for the sensors prototyping stage and simulation of its viability for force and deformation measurement that can find applicability not only in SHM monitoring but in a variety of situation where deformations have to be registered.

The main aim of this investigation is to investigate light guide based optical device concept viability. In order to complete the task raised in our aim we need to:

1) Build light guide based optical device theoretical model.

2) Based on theoretical model, perform optical simulations in order to collect data for devices response affected under different external forces.
3) Review simulation results.

4) Based on recommendations from simulation results review prepare light guide based force and deformation device functional prototype sample.

\section{Theoretical design considerations}

In the simplest optical systems that are used to guide light from one point in the system to the other, passing through materials or mediums with different optical properties it is essential to know the materials refraction index. By knowing these values in addition to source light incidence angle it becomes possible to predict direction of the outgoing light (or guide the light in direction that is required) after it passes the boundary between two different mediums. This can be calculated by applying Snell's Law to the optical system under investigation:

$$
n_{1} \sin \left(\theta_{1}\right)=n_{2} \sin \left(\theta_{2}\right),
$$

here $n_{1}$ and $n_{2}$ are refraction indices of medium that light is originating from and of medium from which light is exiting, respectively and knowing the sinus of incident light $\theta_{1}$ angle, the refracted light angle $\theta_{2}$ can be calculated (Fig. 1).

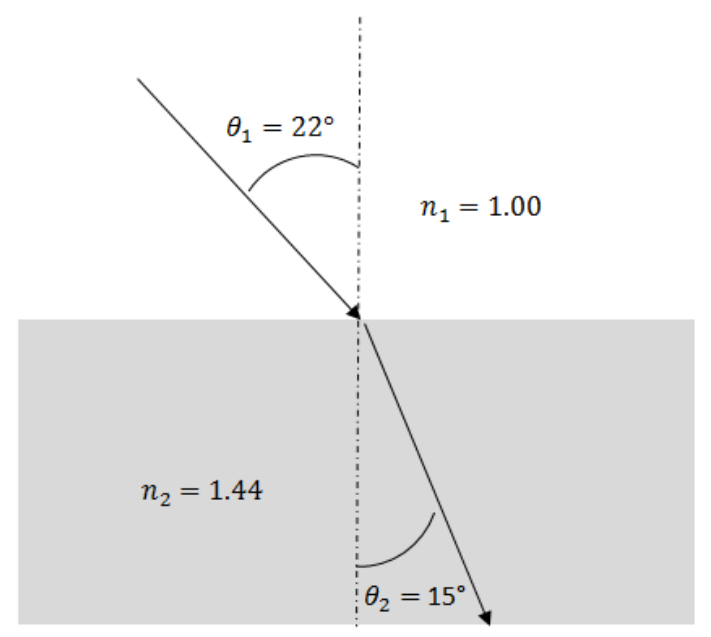

Fig. 1 Graphical representation of Snell's Law

The light guide optical device in sense is a simple optical system with source that emits light that passes through minimum of two mediums. From design point of view Snell's Law enables to define light guide geometry based on how it is necessary to direct the light inside the device's light guide and where the output surface is defined.

As Snell's Law enables us to define the device's light guide geometry, it is necessary to have a starting point that is when the system is in equilibrium, input of light energy into the light guide is equal to the output energy from the light guide. This can be achieved by designing the light guide so that the incident light would have a total internal reflection inside the light guide.

Total internal reflection (TIR) is an optical phenomenon when light travels from material (medium) with lower optical density to a secondary material (medium) with higher optical density at a light incidence angle greater than specific critical angle, then refraction becomes equal to $90^{\circ}$ and theoretically all light energy is reflected at the boundary surface, none is transmitted: 


$$
\theta_{c}=\arcsin \frac{n_{2}}{n_{1}}
$$

where $\theta_{c}$ the critical angle that is sought and $n_{1}$ and $n_{2}$ is are refraction indexes of medium light is originating and of medium light is exiting from respectfully.

By applying TIR and Snell's Law, possible light guide design, for our application, can be evaluated (Fig. 2). The light guide design here is defined so, that at starting conditions there exist a critical angle (TIR condition) for incidence light at the air-light guide interface. Under such conditions incidence light from the source, directed at the light guide input surface, would hit the air-light guide interface at critical angle - all of the light would be reflected back inside the light guide in the direction of the output surface. And as the light guide would be deformed from its starting conditions due to application of some outside force the angle at which light from the source hits the air-light guide interface would change (decreasing or increasing critical angle) - leading to a part of the incidence light being reflected inside the light guide and part being transmitted through the air-light guide interface, the input and output light energy ratio would change accordingly to the change of incidence light angle at which it hits the air-light guide interface.

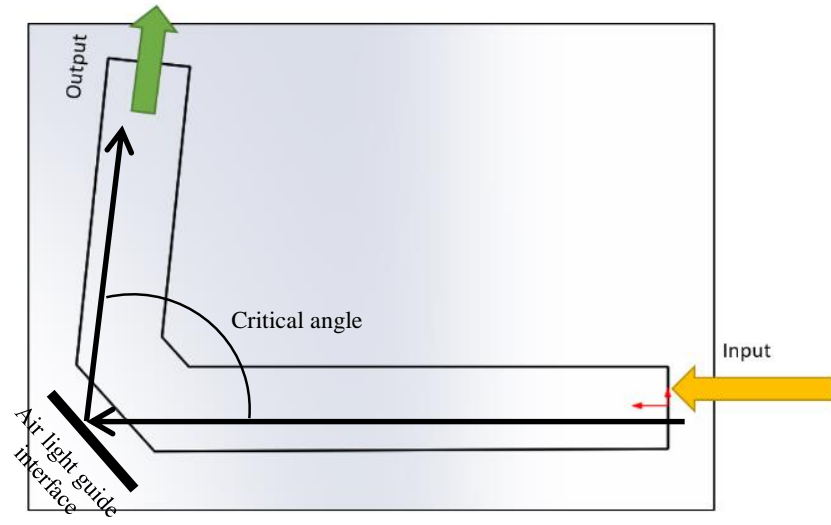

Fig. 2 Possible light guide design at starting conditions

\section{Simulation setup}

Based on theoretical investigation it is necessary to define the geometry of the light guide, it is known that the starting point has to be such, that the incidence light from the source would have incidence angle inside the light guide equal to critical angle for total internal reflection to take place and for the system to be in equilibrium. The light guide material will be used Poly (methyl methacrylate (PMMA)) due to its low optical density, good impact strength and its transmissivity (transmits up to $92 \%$ of visible light in the wavelength range of $400 \mathrm{~nm}$ and $1100 \mathrm{~nm}$ (for $3 \mathrm{~mm}$ thickness sheet)). Light guide bending angle was set equal to $42.8^{\circ}$, a critical angle for air/PMMA boundary interface.

Missing boundary conditions for the simulation of light guide optical device are light source, light detector and the geometry of the light guide itself.

For light source a vertical-cavity surface-emitting laser OPV $302(880 \mathrm{~nm})$ produced by Optek Technology (Fig 3,4) has been defined.

OPV 302 VCSEL has been chosen since it is a widely available coherent light source. Having one wave- length light source should reduce and remove additional uncontrollable items.

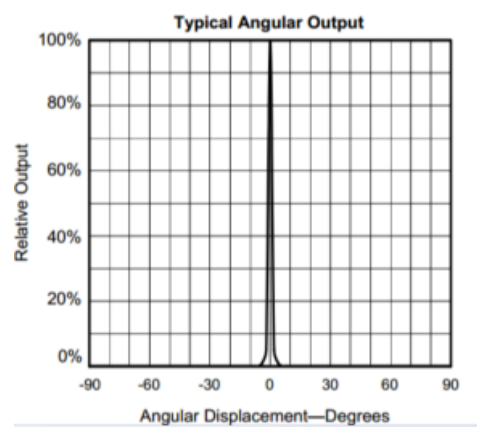

Fig. 3 OPV 302 VCSEL angular output

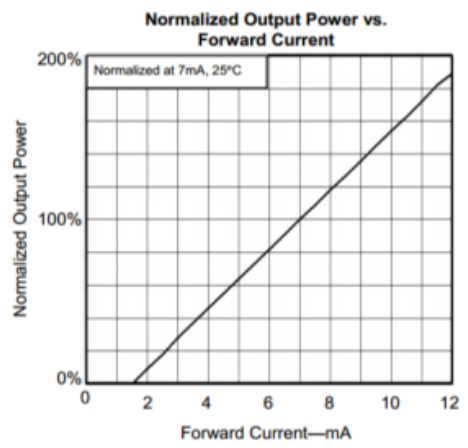

Fig. 4 OPV 302 VCSEL normalized power output

As the light source has been defined next light detector has to be chosen. Due to type of the light source, PIN photodiode Vishay WBPW34S, max. sensitivity in the range of $940 \mathrm{~nm}$ has been defined as light detector. (Figs. 5, 6)

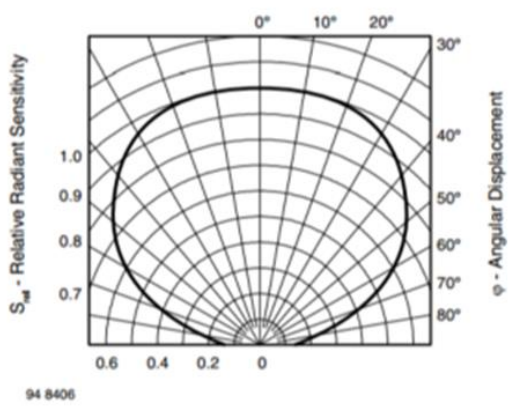

Fig. 5 WBPW34S photodiode angular characteristics

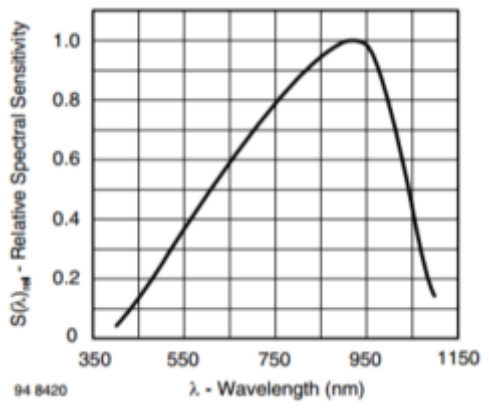

Fig. 6 WBPW34S photodiode spectral characteristics

WBPW34S photodiode has been chosen to have peek sensitivity in the range of OPV 302 output wavelength and due to wide market availability.

As the components for light guide optical device 
simulation have been defined what is left is to choose necessary layout of light source and detector with respect to light guide. The aim here is to have incident light beam focused in the light guide. To generate the CAD model of the light guide for optical device CAD Solidworks 2014 software package has been used.

As the CAD (Fig. 7) model has been constructed.

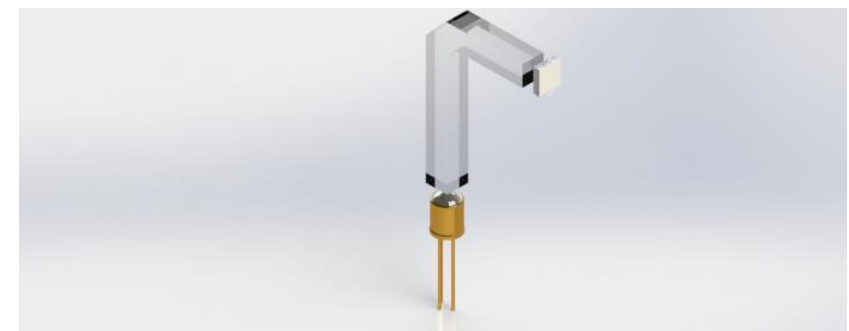

Fig. 7 Light guide based optical device CAD model

The light guide based optical device model has to be evaluated using physical simulations software package TracePro Standard 7.3.7. Inside the simulation tool the starting conditions for the simulations were defined as follows:

1) Light guide material: Poly (methyl methacrylate (PMMA)).

2) Environment medium: Air $\left(+25^{\circ} \mathrm{C}\right)$.

3) Light source power output: $10 \mathrm{~W} / \mathrm{m}^{2}$.

4) Number of light rays: 10000 .

Total - Irradiance Map for Absorbed Flux

Assy_42.5deg|NAUO3\#Photodiode;Boss-Extrude15 Surface 4 Global Coordinates
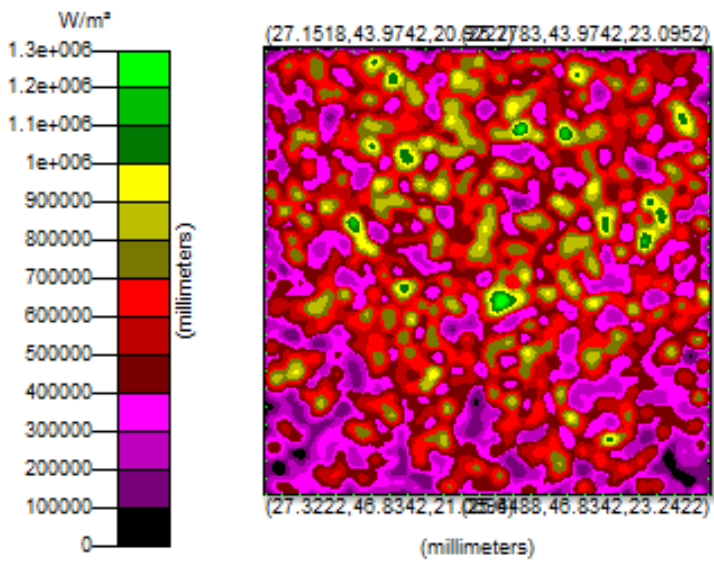

Min:39134, Max: 1.2795e+006, Ave:5.0339e+005

Total Flux:4.1428 W. Flux.Emitted Flux:0.41428, 5402 Incident Rays

Fig. 8 Light guide flux output at $43^{\circ}$ bending angle

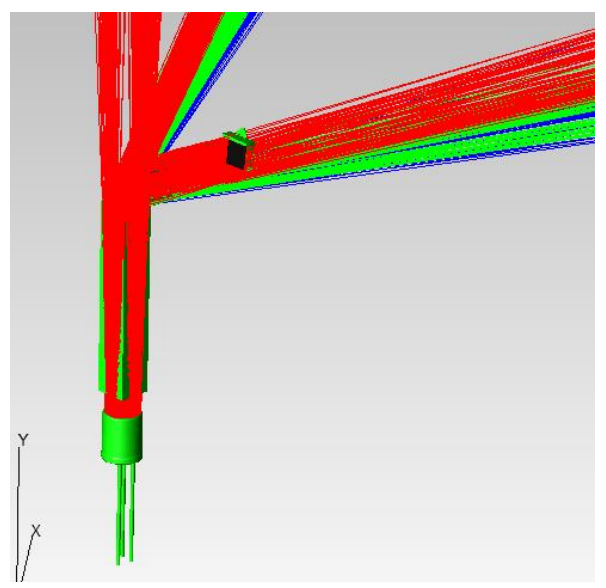

Fig. 9 Light guide model view at $43^{\circ}$ bending angle

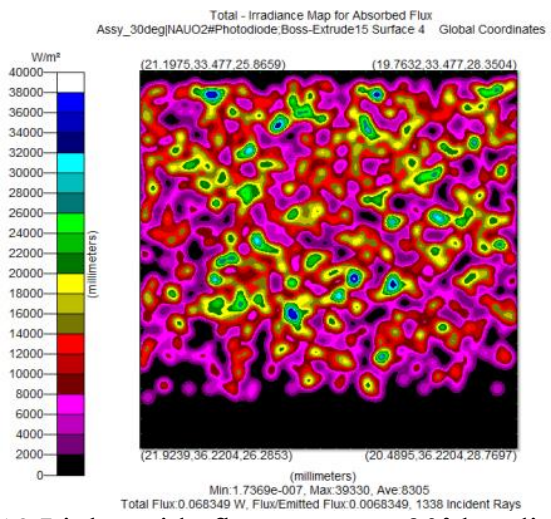

Fig. 10 Light guide flux output at $30^{\circ}$ bending angle

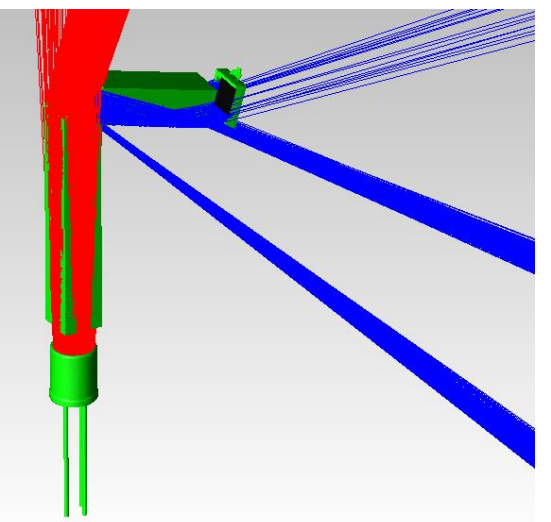

Fig. 11 Light guide model view at $30^{\circ}$ bending angle

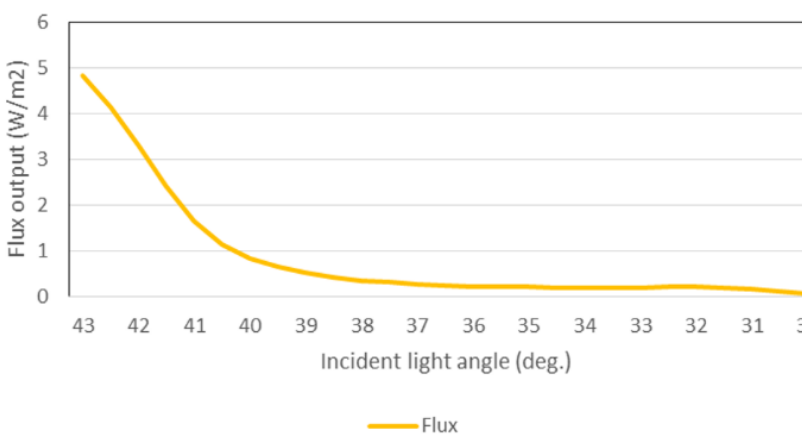

Fig. 12 Light guide optical simulation results

In order to evaluate how the input/output light ratio changes in the light guide it has been deflected from critical angle of $43^{\circ}$ (Figs. 8, 9) to $30^{\circ}$ (Figs. 10, 11) at $0.5^{\circ}$ step. This reduction in critical angle has to simulate deflection of the light guide under effect of outside forces. For each simulation step, irradiance map and flux output from the light guide onto the detector together with flux input/output ratio has been recorded. Simulation results have been collected and can be seen in (Fig. 12).

\section{Recommendations for prototype development}

From our simulation results the following list of items has to be taken into consideration when developing functional prototypes of light guide optical derive:

1) Starting point of light cover design should be calculation of critical angle based on the material and environment medium refraction indexes used for the prototype. 
2) Coherent light source should be used as a source to replicate the simulation results.

3) Distance from light source to the light guide should be adjusted so that the light beam would be focused on the critical angle surface.

4) Light guide should be designed so that to expect deflection is in the range of $1^{\circ} \div 2^{\circ}$ from set critical angle.

\section{Functional light guide based force and deflection measurement prototype device assembly}

Based on recommendations after reviewing optical simulation results, first functional light guide based force and deformation measurement device has been assembled (Fig. 13).

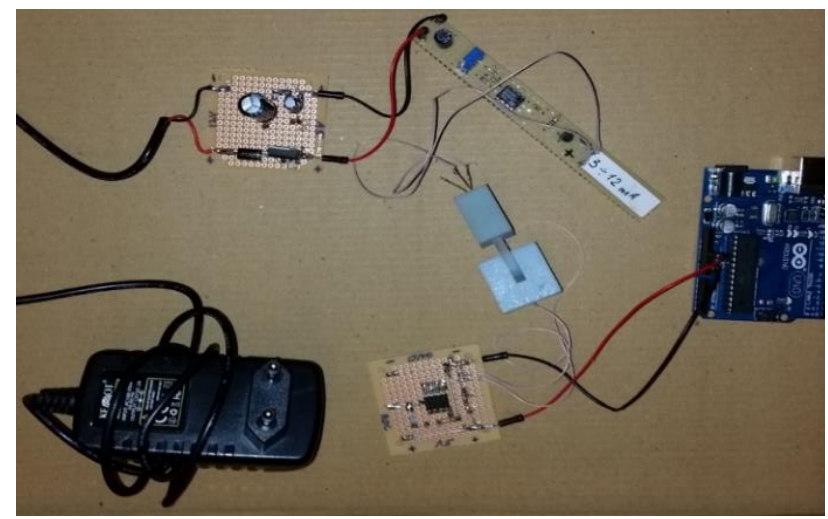

Fig. 13 Light guide based force and deformation measurement device prototype

The electrical schematics used for light guide based force and deformation measurement device prototype are given in (Fig. 14).
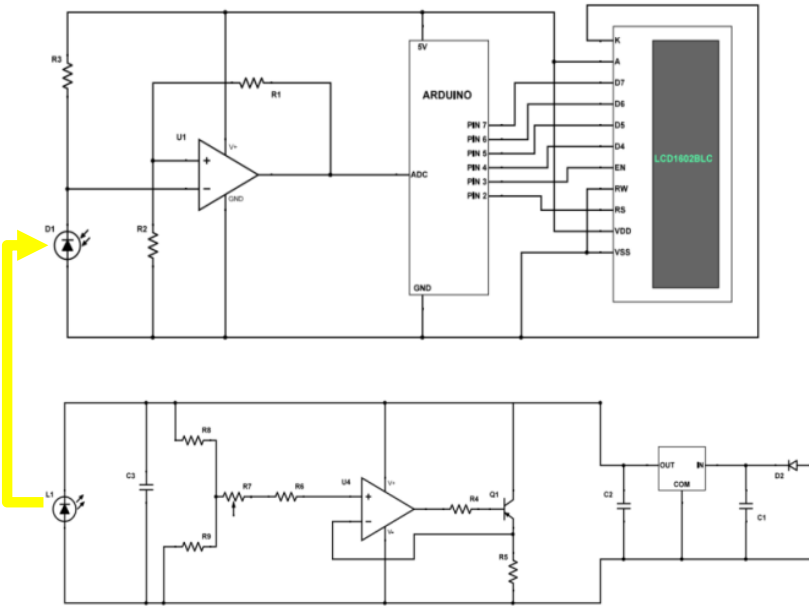

Fig. 14 Light guide based force and deformation device prototype electrical schematics

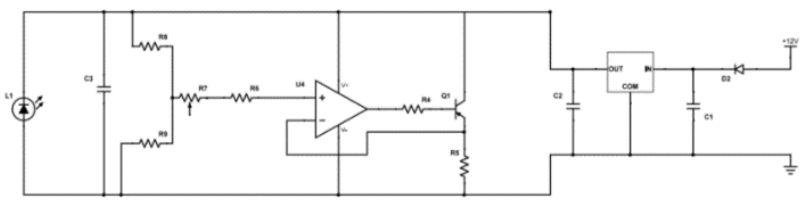

Fig. 15 Light guide based force and deformation device power supply for VCSEL OPV302 schematics
The electrical schematics of the prototype device (Fig. 14) can be split into three parts. The power supply line with constant voltage (5V) and variable current (3-12mA) for the VCSEL OPV302 power supply (Fig. 15). Electrical components used for power supply of VCEL OPV302 are presented in Table 1.

Table 1

Electrical components used for power supply of VCSEL OPV302

\begin{tabular}{|c|c|}
\hline Symbol & Value \\
\hline D2 & Schottky diode 60V3A DO201AD \\
\hline C1 & Electrolytic capacitor 220uF 63V +20/-10\% \\
\hline C2 & Electrolytic capacitor 47uF 25V \\
\hline $\begin{array}{c}\text { OUT IN } \\
\text { COM }\end{array}$ & Positive Voltage Regulator 7805 TO220 \\
\hline Q1 & Amplifier transistor C547B \\
\hline R4 & Resistor 510 $\Omega$ \\
\hline R5 & Resistor $51 \Omega$ \\
\hline U4 & Operational amplifier LM2904N \\
\hline R6 & Resistor 510 $\Omega$ \\
\hline R7 & Trimmer potentiometer Bochen 3296 100K \\
\hline R8 & Resistor 3,76 K $\Omega$ \\
\hline R9 & Resistor 344 $\Omega$ \\
\hline C3 & OPV 302 VCSEL \\
\hline L1 & \\
\hline
\end{tabular}

Photodiode power supply and signal amplification (5 times) circuit (Fig. 16). Electrical components used for this circuit part are presented in Table 2.

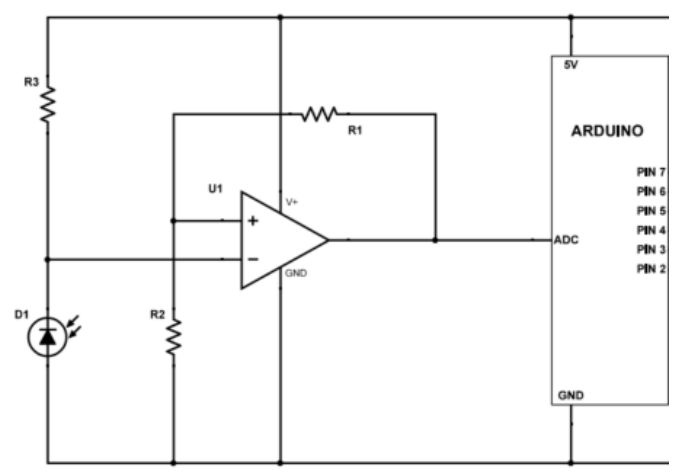

Fig. 16 Photodiode power supply and signal amplification circuit

Table 2

Photodiode power supply and signal amplification circuit electrical components

\begin{tabular}{|c|c|}
\hline Symbol & Value \\
\hline R1 & Resistor $109,5 \mathrm{~K} \Omega$ \\
\hline R2 & Resistor $21,8 \mathrm{~K} \Omega$ \\
\hline R3 & Resistor $1 \mathrm{M} \Omega$ \\
\hline U1 & Dual Operational Amplifier LM358N \\
\hline D1 & Photodiode PD15-22b \\
\hline
\end{tabular}

Arduino programmable microcontroller board with LCD screen for measurement data output (Fig. 17).

Functional light guide based force and deformation measurement prototype device can be used for further investigation to confirm the simulation results. 


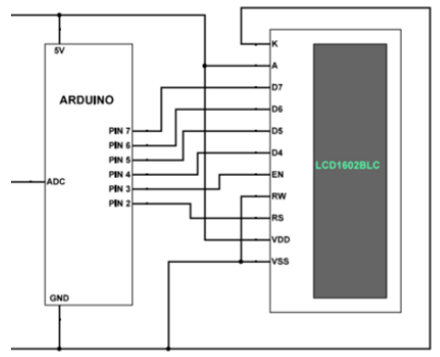

Fig. 17 Arduino programmable microcontroller board with LCD screen

\section{Acknowledgments}

This work has been funded by a grant (No. SEN10/15) from the Research Council of Lithuania. Project acronym: "CaSpine".

\section{References}

1. Zhanga, L.; Luoa, Y.; Taoa, F.; Hu Lia, B.; Rena, L.; Zhanga, X.; Guoa, H., Chenga, Y., Hua, A.; Liua, Y. 2013. Cloud manufacturing: a new manufacturing paradigm, Enterprise Information Systems 1-21, iFirst article. http://dx.doi.org/10.1080/17517575.2012.683812.

2. Ostasevicius, V.; Jurenas, V.; Markevicius, V; Gaidys, R.; Zilys, M.; Cepenas, M.; Kazlauskiene, L. 2015. Self-powering wireless devices for cloud manufacturing applications, Int J Adv Manuf Technol 83(9): 1937-1950.

http://dx.doi.org/10.1007/s00170-015-7617-x.

3. Sasayama, T.; Oho, S.; Kuroiwa, H.; Suzuki, S 1987. Recent developments of optical fiber sensors for automotive use, Fiber and Integrated Optics 7: 255-271. http://dx.doi.org/10.1080/01468038808221282.

4. Parro, T. 1989. Fiber Optic Sensors for Industrial Applications Fiber Optic Sensors for Industrial Applications, ISA Transactions 28(2): 31-34. http://dx.doi.org/10.1016/0019-0578(89)90038-4.

5. Inaudi, D. 2000. Application of civil structural monitoring in Europe using fiber optic sensors, Prog. Struct. Engng Mater. 2: 351-358. http://dx.doi.org/10.1002/15282716(200007/09)2:3<351::AID-PSE41>3.0.CO;2-5.

6. Guo, H.; Xiao, G.; Mrad, N.; Yao, J. 2011. Fiber optic sensors for structural halth monitoring of air platforms, Sensors 11(4): 3687-3705. http://dx.doi.org/10.3390/s110403687.

7. Udd, E.; Spillman, W.B. 2011. Fiber Optic Sensors An Introduction for Engineers and Scientists. UK: A John Willey \& Sons, Inc. 511p.

8. Culshaw, B.; Kersey, A. 2008. Fiber-Optic Sensing: A Historical Perspective, Journal of lightwave technology 26(9): 1064-1078. http://dx.doi.org/10.1109/JLT.0082.921915

9. US Department of Transportation, Federal Highway Administration. 2013. Material Type of Structure by State National Bridge Inventory [online] NBI [accessed 20 March 2015]. Available from Internet: http://fhwa.dot.gov/bridge/material.cfm.

10. Abdel-Jaber, H.; Glisic, B. 2014. A Method for the onsite determination of prestressing forces using longgauge fiber optic strain sensor, Smart Materials and
Structures 23(7): 1-9.

http://dx.doi.org/10.1088/0964-1726/23/7/075004.

11. Pieraccini, M.; Fratini, M.; Parrini, F.; Macaluso, G.; Atzeni, C. 2004. High-speed CW step-frequency coherent radar for dynamic monitoring of civil engineering structures, Electronic letters 40(14): 907-908. http://dx.doi.org/10.1049/el:20040549.

12. Huang, S.; Chang, J.; Qin L.; Yang, X.; Li, Z.; Cheng, X. 2011. A cement based piezoelectric composite sensor for health monitoring of civil engineering structure, Advanced Materials Research 306-307: 293296. http://dx.doi.org/10.4028/www.scientific.net/AMR.306-307.293.

13. Abdulfatah, A.A.G.; Arsad, N.; Reaz, M.I.; Asharif, A.; Bakar, A. 2014. Advances in Bio-Tactile Sensors for Minilally Invasive Surgery Using the Fiber Bragg Grating Force Sensor Technique: A Survey, Sensors 14(4): 6633-6665.

http://dx.doi.org/10.3390/s140406633.

14. Taffoni, F.; Formica, D.; Saccomandi, P.; Di Pino, G.; Schena, E. 2013 Optical Fiber-Based MRCompatible Sensors for Medical Applications: An Overview, Sensors 13: 114105-14120. http://dx.doi.org/10.3390/s131014105.

V. Ostaševičius, P. Karpavičius, G. Janušas, G. Balevičius

DESIGN OF UNIFORM LIGHT GUIDE BASED, FORCE AND DEFLECTION MEASUREMENT PROTOTYPE DEVICE FOR CONSTRUCTION OR BIOMEDICINE APPLICATION

$\mathrm{S}$ u m m a r y

This paper presents the investigation prepared in order to verify light guide based optical sensor for force and deformation measurements. Based on the results obtained during the whole period of investigation, design of prototype sample for light guide based optical sensor has to be approved, improvements based on the received results included.

In order to define design (geometry) a theoretical design model has been created based on Snell's Law and Total Internal Reflection phenomenon. Based on the output from the theoretical investigation, a CAD model has been created. This virtual model of the sensor has been used to perform optical simulations in wide range of light guide deflection angles, to record flux changes with decreasing the deflection angle from critical (TIR) to $30^{\circ}$ by $0.5^{\circ}$ step.

After simulation is completed by reviewing simulation results we can confirm that the light guide based optical sensor for force and deflection measurements has linear output flux correlation with changing incidence angle in the range of $1^{\circ}-2^{\circ}$.

For further investigation to confirm correlation between simulation and measurement results the light guide based force and deformation measurement functional prototype device has been assembled.

Keywords: light guide, optical sensor, fiber optics.

Received June 27, 2016 Accepted August 01, 2016 